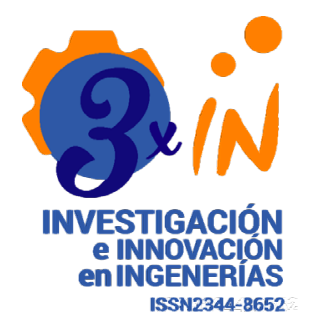

OPEN ACCESS

Recibido: 10/08/2021

Aceptado: 08/11/2021

Publicado: 07/02/2022

Correspondencia de autores: gabriel.castiblanco@unimilitar.edu.co

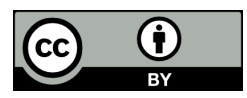

Copyrith 2020 by Investigación e Innovación en Ingenierías

\section{Espectroscopía de impedancia electroquímica, herramienta para la determinación de fallas e imperfecciones en recubrimientos internos de tuberías}

\section{Electrochemical impedance spectroscopy, tool for determining flaws and imperfections in internal coatings of pipes}

\author{
Gabriel Humberto Castiblanco (iD) Willian Aperador Chaparro
}

Universidad Militar Nueva Granada, Colombia

Resumen

Objetivo: Mostrar los resultados obtenidos de las pruebas de espectroscopia de impedancias realizadas a recubrimientos $\mathrm{FBE}$ y líquidos utilizados en tuberías recubiertas internamente que transportan fluidos altamente corrosivos del sector petrolero. Metodología: El estudio fue realizado mediante pruebas de laboratorio con el equipo Gamry 3000 y equipo prototipo, efectuando la medición a probetas recubiertas con resina epóxica en polvo Resicoat HMH09QF y recubrimiento líquido SigmaShield 1200, que fueron sometidas a procesos de soldadura que generaron diferentes fallas como los son ampollas, grietas, fisuras y quemaduras que son el objeto de análisis y medición de impedancias para lograr una caracterización y determinación de valores que permitan clasificar estos problemas con el fin de minimizarlos y corregirlos para evitar accidentes por fugas debido a fallas por procesos de alta corrosión en tuberías, mitigando el impacto ambiental. Resultados: Con los ensayos realizados a las probetas recubiertas se evidencio que las fallas en donde el recubrimiento se quema o se desprende de la tubería se presentan impedancias del orden de x104 y x105, para los casos de recubrimiento sin fallas las impedancias están en el orden de x109 y superiores, dejando las fallas intermedias como fisuras y ampollas en valores intermedios del orden de x108. Conclusiones: $\mathrm{El}$ estudio permitió la caracterización de las diferentes fallas en los recubrimientos internos de tuberías que ocurren en los procesos de soldadura de las juntas, obteniendo valores de impedancia para cada una de las fallas que se pueden presentar y finalmente asociando unos rangos entre los que se caracterizan los diferentes problemas y fallas de los recubrimientos.

Palabras clave: Corrosión, Espectroscopia de impedancia (EIS), FBE, Gamry Echem analyst, Sleeve.

Abstract

Objective: To show the results obtained from impedance spectroscopy tests performed on FBE coatings and the liquids used in pipelines internally coated that carry highly corrosive fluids in the oil industry. Methodology: This study is carried out by laboratory tests with Gamry 3000 equipment and prototype equipment, measuring pieces of test tubes coated with Resicoat HMH09QF epoxy resin powder and SigmaShield 1200 liquid coating. These pieces are subject to welding processes that generated different failures such as blisters, cracks, fissures, and burns. They are the object of analysis and measurement of impedances to achieve a characterization and determination of values to classify these problems minimizing and correcting them, to avoid accidents caused by leaks due to high corrosion failures in the pipes' processes, mitigating the environmental impact. Results: The tests carried out on the coated pieces evidence that failures such as coating burns or detaches from the pipeline, the impedances are in the order of x104 and x105. On the contrary, in the coating cases without failures, the impedances are x109 and higher. The intermediate failures such as cracks and blisters in intermediate values are in the order of $x 108$. Conclusions: TThe study allowed the characterization of different failures in the internal pipeline coatings that appear in weld joints. It was possible to obtain impedance values for each coating failure, and finally, it was possible to associate them into ranges according to their problems and coating failures.

Keywords: Corrosion, FBE, Gamry Echem analyst, impedance spectroscopy (EIS), Sleeve.

Como citar (IEEE): G. Humberto-Castiblanco., W. Aperador-Chaparro. “Espectroscopía de impedancia electroquímica, herramienta para la determinación de fallas e imperfecciones en recubrimientos internos de tuberías". Investigación e Innovación en Ingenierías , vol. 10, $n^{\circ} 1,07-16,2022$. DOI: https://doi.org/10.17081/invinno.10.1.5668 


\section{Introducción}

Actualmente en la industria petrolera se aplican recubrimientos internos a las tuberías de transporte de gas, agua y crudo para disminuir los procesos corrosivos debidos a las composiciones químicas con alta salinidad (cloruro de sodio) entre (7000-15000) ppm [1], que aumentan la posibilidad de formación de incrustaciones de carbonato de calcio en las paredes de las tuberías, se tiene también parámetros críticos que aumentan los factores de corrosión entre estos se encuentra $\mathrm{CO}_{2}(30-40) \mathrm{ppm}$, oxígeno disuelto (OD) (3-6) ppm, $\mathrm{H}_{2} \mathrm{~S}$ (0.2-5) ppm, este es producido por la acción metabólica de bacterias sulfato reductoras, también hay sólidos en suspensión (hierro con contenidos entre 4-20 ppm), entre otros componentes, que se encuentran por fuera de los límites establecidos en las normas Nace y ASTM 4412-02 [2]. Las características de los fluidos mencionadas anteriormente han generado la necesidad de aplicar recubrimientos internos que funcionen como barreras que eviten el contacto directo entre los fluidos y las superficies metálicas de las tuberías, para evitar las altas tasas de corrosión, este tipo de recubrimientos posee pigmentos anticorrosivos, compuestos por químicos que generan barreras de protección que pueden ser de dos tipos, de pasivación anódica o catódica o por algún componente menos noble que el hierro [3,4,5,6].

Los recubrimientos analizados en las pruebas son de doble naturaleza una de tipo líquido compuesta principalmente de una base epóxica, alquídica o un uretano que se mezclan con un disolvente para permitir la reacción de secado, siendo el más utilizado el epóxico, este tipo puede aplicarse en una capa, dos capas o múltiples capas, dependiendo del espesor de película que requiera el recubrimiento acorde a la fábrica o en función del tiempo de vida para el que se proyecte el diseño y la selección, algunas de las ventajas que caracterizan a estos recubrimientos, es el bajo costo y la facilidad de aplicación ya que se puede realizar de forma manual con equipos aspersores [7]. Son una buena alternativa para sistemas que manejan bajas presiones (285-1480) psi correspondientes respectivamente a las designaciones ANSI 150 - 600 que son las más utilizadas en procesos de transporte de crudo y gas con temperaturas moderadas no superiores a los $200^{\circ} \mathrm{F}$, una de las desventajas es que no son amigables con el medio ambiente y son perjudiciales para la salud debido al alto contenido particulado que se genera al momento de su aplicación, provocando problemas respiratorios a los operarios, otra desventaja relevante es el alto tiempo de curado que puede ser de hasta 3 días [8]. El otro tipo de recubrimiento utilizado es el epóxico adherido por fusión (FBE): (FUSION-BONDED EPOXY), son una mejor alternativa en los casos donde se cuenta con temperaturas superiores a los $200 \mathrm{~F}$ y presiones superiores a los 1500 Psi, entre los que se tienen las clases ANSI 900, 1500 y 2500 que son las más utilizadas para los procesos de inyección de agua, para su aplicación se requiere elevar las piezas a recubrir a temperaturas cercanas a los $250 \mathrm{~F}$, por esto se requiere de hornos para lograr este propósito, posteriormente lo que se realiza es la aplicación de la pintura que viene en polvo y es fundida con las piezas al contacto de ahí que estos recubrimientos poseen una mayor adherencia, mejor acabado superficial y su secado es realizado de forma progresiva en el horno con tiempos muy cortos que no exceden las dos horas, si comparamos los recubrimientos FBE y los líquidos estos son más amigables con el medio ambiente ya que disminuyen los contenidos particulados y por ser fundidos disminuyen el riesgo respiratorio de los operarios $[9,10]$.

Basados en los dos tipos de recubrimientos y utilizando la técnica o método electroquímico enfocado en encontrar el valor de la impedancia para las diferentes fallas que se producen en los procesos de soldadura de tuberías recubiertas internamente se utilizan señales sinusoidales en un rango de frecuencias entre $0.03 \mathrm{~Hz}$ hasta $20 \mathrm{~Hz}$ con el fin de caracterizar y probar los recubrimientos encontrando fallas como lo son: desprendimiento del recubrimiento, recubrimiento quemado, ampollas (burbujas en la pintura), grietas, fisuras y porosidades que generan absorción de agua que incrementa las velocidades de corrosión y falla de la integridad en las tuberías. 


\section{Metodología}

La caracterización de las fallas en rangos de impedancia permite definir puntos de prueba que eviten o mitiguen problemas de rupturas y derrames que causan pérdidas millonarias y problemas ambientales, que a su vez pueden llegar a comprometer las comunidades donde son instalados los sistemas de transporte.

\section{Configuración de equipos, preparación de probetas y recubrimientos utilizados}

Para el desarrollo de las pruebas se utilizaron dos equipos un prototipo desarrollado para viajar por dentro de la tubería y realizar las mediciones directamente en juntas soldadas. Este equipo cuenta con un generador de ondas sinusoidales AD9850 con variación de frecuencias desde $0.03 \mathrm{~Hz}$ hasta $100 \mathrm{kHz}$ y sistema de adquisición de datos mediante tarjetas STM32F446RE y plataforma de datos en Matlab [11]. El otro equipo es un Gamry 3000 [36], que es un potenciostato / galvanostato / ZRA de alto rendimiento para pruebas electroquímicas. En ambos sistemas se utilizó un electrodo de referencia $\mathrm{RE}-5 \mathrm{~B} \mathrm{Ag} / \mathrm{AgCl}(3 \mathrm{M} \mathrm{NaCl}$ y un contraelectrodo de platino MW-1032 BASI, ensamblados en una celda fabricada en teflón. En la Figura 1 , se muestra un esquema general de disposición de los electrodos y su distribución de la configuración del sistema de medición con el equipo Gamry y en la Figura 2, se observa la disposición que se tiene en el sistema o celda con el prototipo.

Figura 1. Configuración de los electrodos usados en el sistema de medición el equipo Gamry 3000.

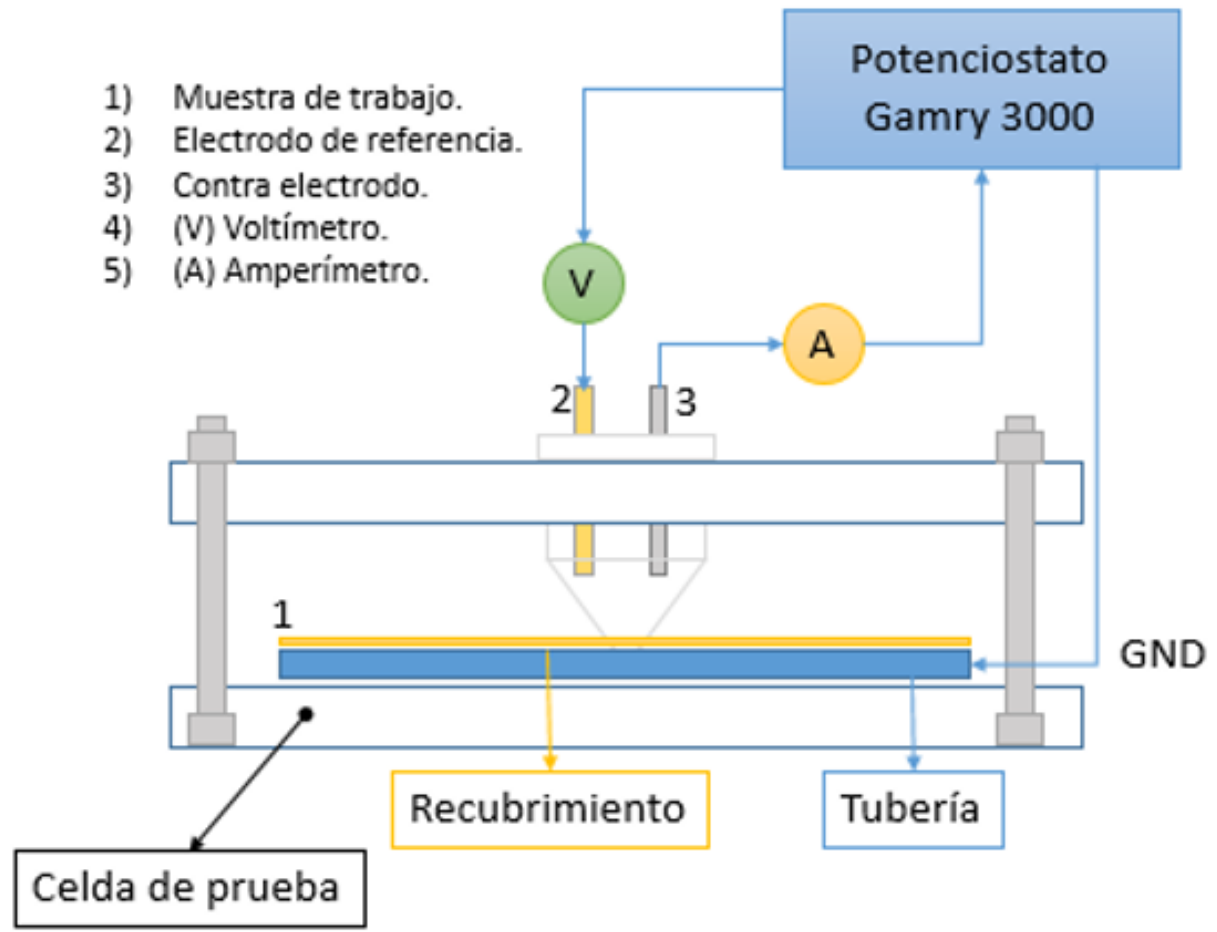

Fuente: Elaboración propia. 
Figura 2. Configuración de los electrodos usados en el sistema de medición con el prototipo desarrollado.

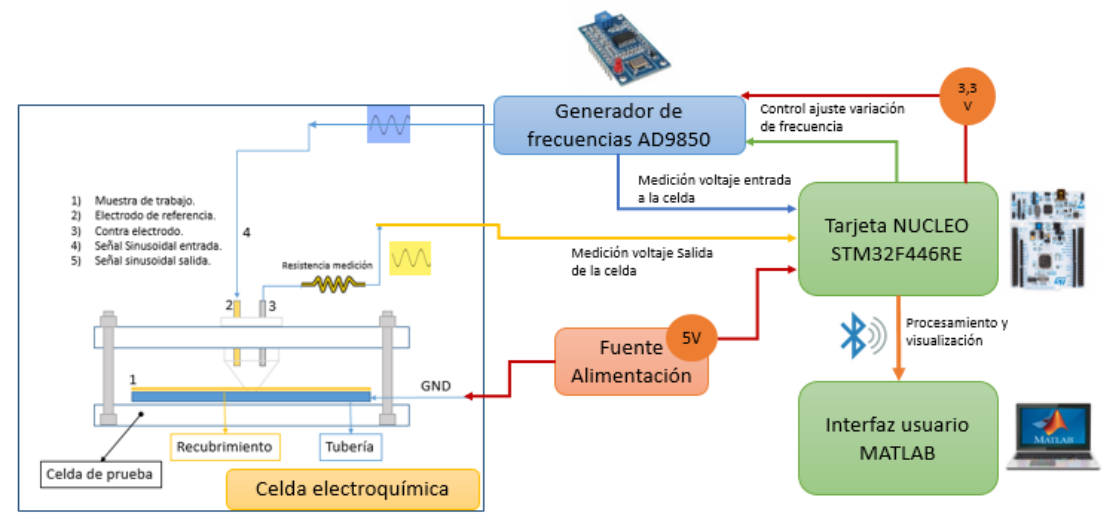

Fuente: Elaboración propia.

Los recubrimientos utilizados en la evaluación, análisis y caracterización de fueron el SigmaShield 1200 [11] de PPG y el Resicoat HMH09QF [12] de AkzoNobel [13].

La preparación de las probetas se realizó teniendo en cuenta las recomendaciones de los proveedores, en el proceso donde se realizaron los siguientes pasos:

- Corte de probetas de acero carbono ASTM A105 de 10cmX10cm [13].

- Preparación superficial con limpieza SSPC-SP5 mediante proceso de granallado, obteniendo los respectivos perfiles de anclaje de 2.5 mils.

- Aplicación del recubrimiento SigmaShield 1200 mediante pistola de aplicación con boquilla de 0.53 $\mathrm{mm}$ a temperatura de $20^{\circ} \mathrm{C}$ y una presión de $30 \mathrm{MPa}$ (4350 psi). Posteriormente se dejaron en proceso de curado durante una semana.

- Aplicación del recubrimiento Resicoat HMH09QF, aumento de temperatura de las probetas en horno a $204^{\circ} \mathrm{C}$ y aplicación con pistola de boquilla de $0.53 \mathrm{~mm}$ fluidizando mediante calentador el polvo epóxico a $204^{\circ} \mathrm{C}$. Se realiza proceso de curado a $218^{\circ} \mathrm{C}$ durante una hora y posterior enfriamiento.

- Finalizado el proceso de aplicación se verificaron visualmente los recubrimientos y se realizó una prueba de Holiday (medición de discontinuidades), garantizando que la aplicación fuera adecuada.

Luego de tener las probetas recubiertas se realizó proceso de soldadura entre dos probetas con el mismo recubrimiento, con el propósito de generar las respectivas fallas que se presentan normalmente en los procesos de instalación y de esta forma caracterizar estos problemas con la técnica EIS $[14,15,16]$.

Algunas de las fallas más comunes que se presentan en el proceso de soldadura son: discontinuidades, fisuras, burbujas en la pintura, desprendimientos y quemaduras en los recubrimientos, en las Figura 3 y Figura 4 se pueden observar estos problemas. 
Espectroscopía de impedancia electroquímica, herramienta para la determinación de fallas e imperfecciones en recubrimientos internos de tuberías

Figura 3. Fallas probeta recubierta con SigmaShield 1200 soldada. Fotografía tomada a probetas obtenidas y soldadas.

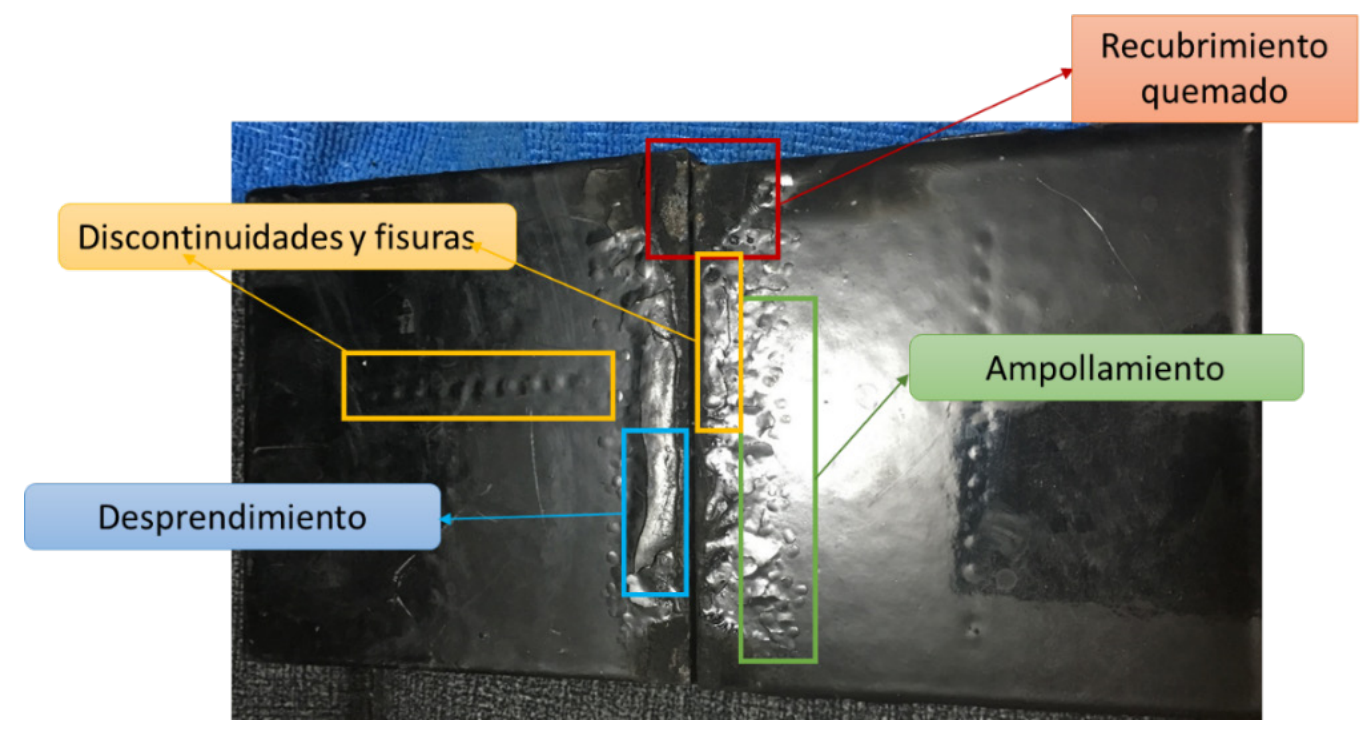

Fuente: Elaboración propia.

Figura 4. Fallas probeta recubierta con Resicoat HMH09QF soldada. Fotografía tomada a probetas obtenidas y soldadas.

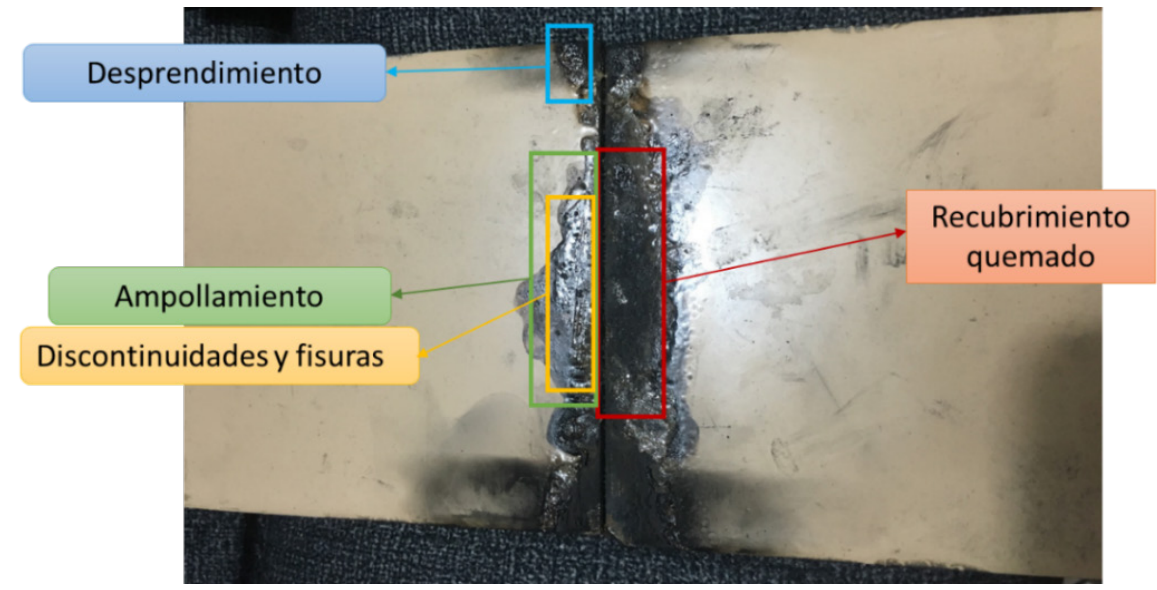

Fuente: Elaboraqción propia.

Para facilitar los ensayos se realizó un corte de las probetas mediante equipo de corte de electroerosión con hilo, dejando las probetas de un tamaño de $5 \mathrm{~cm} \times 10 \mathrm{~cm}$ para facilitar el ensayo de EIS [17].

\section{Calibración del sistema de medición EIS Gamry 3000 y prototipo}

La calibración del equipo Gamry y el prototipo se realizó mediante celda Dummy [14].

Para el proceso de medición de la celda se utilizó una resistencia de $1 \mathrm{k} \Omega$ para acoplar el medidor del prototipo, esta resistencia se requiere para aumentar el voltaje de la medición y tener un buen rango de muestreo, la impedancia de la celda hasta los $0.01 \mathrm{~Hz}$ es de $4257 \Omega$ y en las frecuencias mayores a $100 \mathrm{kHz}$ el valor baja a $1247 \Omega[16]$. 
El proceso de medición realizado con el prototipo y comparados con los valores teóricos de la celda Dummy se encuentran consignados en la Tabla 1 puede observarse que los datos del prototipo presentan un buen comportamiento hasta la frecuencia de $20 \mathrm{~Hz}$, rango que es suficiente para realizar la caracterización de los recubrimientos y los diferentes tipos de fallas.

El rango máximo de error entre la celda Dummy y el prototipo hasta la frecuencia de $20 \mathrm{~Hz}$ fue de $10.38 \%$, estos valores son calculados por medición directa con el prototipo a la celda Dummy y comparados con los valores teóricos de la celda que es un elemento calibrado que permite ajustar los equipos de medición esta celda es usada en los procesos de calibración del Gamry 3000 [17].

Tabla 1. Comparación datos medidos prototipo y teóricos de la celda Dummy

\begin{tabular}{|c|c|c|c|}
\hline $\begin{array}{c}\text { Frecuencia } \\
\left(\mathbf{H z}_{\mathbf{z}}\right.\end{array}$ & $\begin{array}{c}\text { Z medida prototipo } \\
(\boldsymbol{Q})\end{array}$ & Z Teórica Celda Dummy ( $\Omega$ ) & \%error \\
\hline 0.03 & 4443.50 & 4257.00 & $4.38 \%$ \\
\hline 0.06 & 3932.22 & 4257.00 & $7.63 \%$ \\
\hline 0.09 & 4069.99 & 4256.99 & $4.39 \%$ \\
\hline 0.12 & 3847.92 & 4256.99 & $9.61 \%$ \\
\hline 0.15 & 4224.71 & 4256.98 & $0.76 \%$ \\
\hline 0.18 & 4410.69 & 4256.98 & $3.61 \%$ \\
\hline 0.24 & 3996.84 & 4256.96 & $6.11 \%$ \\
\hline 0.3 & 4428.10 & 4256.94 & $4.02 \%$ \\
\hline 0.4 & 4144.05 & 4256.89 & $2.65 \%$ \\
\hline 0.5 & 4068.09 & 4256.83 & $4.43 \%$ \\
\hline 0.6 & 3874.53 & 4256.75 & $8.98 \%$ \\
\hline 0.8 & 4166.06 & 4256.55 & $2.13 \%$ \\
\hline 1 & 4243.97 & 4256.30 & $0.29 \%$ \\
\hline 2 & 4054.14 & 4254.22 & $4.70 \%$ \\
\hline 3 & 3809.62 & 4250.75 & $10.38 \%$ \\
\hline 5 & 4225.60 & 4239.72 & $0.33 \%$ \\
\hline 10 & 4153.95 & 4189.27 & $0.84 \%$ \\
\hline 20 & 4042.93 & 4006.05 & $0.92 \%$ \\
\hline
\end{tabular}

Fuente: Elaboración propia.

\section{Resultados}

El proceso de pruebas del sistema EIS diseñado para el prototipo del proyecto fue realizado en dos (2) fases una inicial correspondiente a la calibración que fue realizada utilizando la celda Dummy del laboratorio usada para la calibración del equipo Gamry 3000 y la segunda fase que correspondió a realizar la medición del recubrimiento FBE y comparar los resultados con los obtenidos en las pruebas de caracterización del equipo Gamry 3000 [18].

Los resultados de las pruebas para cada escenario de falla se muestran en la Tabla 2. 
Tabla 2. Resumen de impedancias obtenidas en las pruebas EIS de laboratorio con Gamry 3000 , en relación exponencial

\begin{tabular}{|c|c|c|}
\hline \multicolumn{2}{|c|}{$\begin{array}{c}\text { Sigmashield 1200 } \\
\text { (liquido) }\end{array}$} & $\begin{array}{c}\text { Resicoat HMH09QF } \\
\text { (FBE) }\end{array}$ \\
\hline Sin fallas & $\mathrm{x} 1010$ & $\mathrm{x} 1010$ \\
\hline $\begin{array}{c}\text { Fallas menores } \\
\text { (Ampollas y fisuras) }\end{array}$ & $\mathrm{x} 108$ & $\mathrm{x} 108$ \\
\hline $\begin{array}{c}\text { Fallas mayores } \\
\text { (Desprendimientos y } \\
\text { quemaduras) }\end{array}$ & $\mathrm{x} 104$ & \\
\hline
\end{tabular}

Fuente: Elaboración propia.

Los resultados de las pruebas realizadas con el prototipo se resumen en la Tabla 3.

Tabla 3. Resumen impedancias obtenidas a las probetas FBE con el prototipo

\begin{tabular}{|c|c|c|c|}
\hline Frecuencia $(\mathrm{Hz})$ & $\mathbf{Z}(\Omega)$ FBE sin fallas & $\mathbf{Z}(\Omega)$ FBE fallas menores & $\mathbf{Z}(\Omega)$ FBE fallas mayores \\
\hline 0.03 & $5.92 \mathrm{E}+09$ & $6.95 \mathrm{E}+08$ & $5.95 \mathrm{E}+05$ \\
\hline 0.06 & $5.76 \mathrm{E}+09$ & $7.37 \mathrm{E}+08$ & $2.74 \mathrm{E}+05$ \\
\hline 0.09 & $5.25 \mathrm{E}+09$ & $6.85 \mathrm{E}+08$ & $3.34 \mathrm{E}+05$ \\
\hline 0.12 & $6.56 \mathrm{E}+09$ & $7.17 \mathrm{E}+08$ & $3.27 \mathrm{E}+05$ \\
\hline 0.15 & $5.70 \mathrm{E}+09$ & $7.17 \mathrm{E}+08$ & $3.75 \mathrm{E}+05$ \\
\hline 0.18 & $5.22 \mathrm{E}+09$ & $8.20 \mathrm{E}+08$ & $3.31 \mathrm{E}+05$ \\
\hline 0.24 & $6.00 \mathrm{E}+09$ & $7.91 \mathrm{E}+08$ & $3.61 \mathrm{E}+05$ \\
\hline 0.3 & $6.42 \mathrm{E}+09$ & $8.61 \mathrm{E}+08$ & $3.87 \mathrm{E}+05$ \\
\hline 0.4 & $4.96 \mathrm{E}+09$ & $6.68 \mathrm{E}+08$ & $2.42 \mathrm{E}+05$ \\
\hline 0.5 & $5.25 \mathrm{E}+09$ & $8.90 \mathrm{E}+08$ & $2.99 \mathrm{E}+05$ \\
\hline 0.6 & $4.96 \mathrm{E}+09$ & $7.36 \mathrm{E}+08$ & $3.17 \mathrm{E}+05$ \\
\hline 0.8 & $6.55 \mathrm{E}+09$ & $9.35 \mathrm{E}+08$ & $2.91 \mathrm{E}+05$ \\
\hline 1 & $5.65 \mathrm{E}+09$ & $8.31 \mathrm{E}+08$ & $3.29 \mathrm{E}+05$ \\
\hline 2 & $5.42 \mathrm{E}+09$ & $6.82 \mathrm{E}+08$ & $3.99 \mathrm{E}+05$ \\
\hline 3 & $4.42 \mathrm{E}+09$ & $8.40 \mathrm{E}+08$ & $2.49 \mathrm{E}+05$ \\
\hline 5 & $6.46 \mathrm{E}+09$ & $7.60 \mathrm{E}+08$ & $3.94 \mathrm{E}+05$ \\
\hline 10 & $4.04 \mathrm{E}+09$ & $7.27 \mathrm{E}+08$ & $2.62 \mathrm{E}+05$ \\
\hline 20 & $5.15 \mathrm{E}+09$ & $8.05 \mathrm{E}+08$ & $2.69 \mathrm{E}+05$ \\
\hline & & & \\
\hline & & & \\
\hline & & & \\
\hline
\end{tabular}

Fuente: Elaboración propia.

Los valores obtenidos de impedancia con el prototipo para el rango posible de frecuencias desde $0.03 \mathrm{~Hz}$ hasta $20 \mathrm{~Hz}$ que es lo permitido con el generador de frecuencias AD9850 generó una respuesta exponencial que presentan el mismo comportamiento de las respuestas obtenidas con el equipo de Gamry, el resumen comparativo puede observarse en la Tabla 4, estos resultados permiten establecer tanto para las pruebas de laboratorio y el prototipo, que recubrimientos bien aplicados presentan comportamientos de impedancias en rangos de $\times 10^{9} \mathrm{o} \times 10^{10}$, recubrimientos con fallas menores en rangos de $\times 10^{8} \mathrm{y}$ recubrimientos con fallas mayores en rangos de $\times 10^{4}$ o $\times 10^{5}$ estos valores de referencia son un parámetro muy importante para poder establecer cuando los recubrimientos utilizados en los sistemas de transporte quedan bien instalados y cuando requieren ser reparados o incluso sustituidos antes de poner en funcionamiento [19,20, 21]. 
Tabla 4. Resumen comparación de impedancias entre prototipo y Gamry en rango exponencial

\begin{tabular}{|c|c|c|}
\hline $\begin{array}{c}\text { Recubrimiento FBE } \\
\text { Resicoat HMH09QF }\end{array}$ & Prototipo & Gamry 3000 \\
\hline Sin fallas & x109 & x1010 \\
\hline $\begin{array}{c}\text { Fallas menores } \\
\text { (Ampollas y fisuras) }\end{array}$ & $\mathrm{x} 108$ & $\mathrm{x}$ x105 \\
\hline $\begin{array}{c}\text { Fallas mayores } \\
\text { (Desprendimientos y } \\
\text { quemaduras) }\end{array}$ & $\mathrm{x} 105$ & \\
\hline
\end{tabular}

Fuente: Elaboración propia.

\section{Conclusiones}

- Los resultados obtenidos nos muestran que los recubrimientos presentan comportamientos de alta impedancia x1010 ohmios para recubrimientos en buen estado, valores de impedancia alrededor de x108 ohmios para recubrimientos con fallas menores, como ampollamientos, fisuras y valores de impedancia x104 y x105 ohmios para recubrimientos con fallas relevantes como desprendimientos y recubrimientos quemados.

- Los valores de impedancia obtenidos nos permiten establecer umbrales característicos en los que las mediciones realizadas a los recubrimientos aplicados en tuberías de transporte pueden considerarse adecuados y en qué momento deben ser reemplazados ya que no cumplen con las necesidades de la aplicación y presentarán fallas en los sistemas.

- En el proceso de pruebas del sistema de medición se encontraron muy buenos resultados de calibración mediante la celda Dummy de Gamry con un error máximo de $10.38 \%$, en el rango de bajas frecuencias hasta $20 \mathrm{~Hz}$, que es un valor suficiente para realizar la medición y caracterización de impedancias en recubrimientos internos de tuberías utilizadas en el sector petrolero.

- El prototipo permite medir adecuadamente el comportamiento y decaimiento de impedancias en los recubrimientos con las diferentes fallas que se presentan en los procesos de soldadura y permite una adecuada caracterización de la existencia de las fallas o la verificación del buen estado del recubrimiento.

\section{Referencias bibliográficas}

1. A. O. Ijaola, P. K. Farayibi, E. Asmatulu, "Superhydrophobic coatings for steel pipeline protection in oil and gas industries: A comprehensive review", Journal of Natural Gas Science and Engineering, vol 83, pp. 103544- 103557, 2020. DOI: https://doi.org/10.1016/j.jngse.2020.103544.

2. A. H. Alamri, "Localized corrosion and mitigation approach of steel materials used in oil and gas pipelines - An overview", Engineering Failure Analysis, vol 116, pp.104735-104762,2020, DOI:https:// doi.org/10.1016/j.engfailanal.2020.104735. 
3. A. A. Fadhil, A. A. Khadom, C. Fu, H. Liu, H. B. Mahood, A. K. Mahmoud, M. Z. Khalaf, A. M. A. Karim, "Ceramics coating materials for corrosion control of crude oil distillation column: Experimental and theoretical studies", Corrosion Science, vol 162, pp. 108220-108235, 2020, https://doi.org/10.1016/j. corsci.2019.108220.

4. R. Abubakirov, M. Yang, N. Khakzad, “A risk-based approach to determination of optimal inspection intervals for buried oil pipelines", Process Safety and Environmental Protection, vol 134, pp. 95-107, 2020, DOI: https://doi.org/10.1016/j.psep.2019.11.031.

5. G. Roa-Rodríguez, W. Aperador, E. Delgado, “Diseño de sistema de monitoreo remoto para evaluación de la corrosión en estructuras de concreto reforzado sometidas a ion cloruro" Ingeniería, Investigación y Tecnología, vol 16, no 4, pp. 565-572, 2015, DOI: https://doi.org/10.1016/j.riit.2015.09.008.

6. F. Bernal Castillo, G. Roa-Rodríguez, C. Cabrera, N. Sierra Melo, W. Aperador, “Determination of the probability and rate of corrosion on reinforced concrete specimens through a remote corrosion monitoring system", Tecciencia, vol. 10, no. 19. pp. 2731 - 2749, 2015, DOI: http://dx.doi.org/10.18180/ tecciencia.2015.19.5

7. A. A. Olajire, "Recent advances on organic coating system technologies for corrosion protection of offshore metallic structures", Journal of Molecular Liquids, vol 269, pp. 572-606, 2018, DOI: https:// doi.org/10.1016/j.molliq.2018.08.053.

8. A. Javadi, H. S. Mehr, M. Sobani, M. D. Soucek, Cure-on-command technology: A review of the current state of the art, Progress in Organic Coatings, vol 100, pp 2-31, 2016, DOI: https://doi.org/10.1016/j. porgcoat.2016.02.014.

9. D.K. Chattopadhyay, K.V.S.N. Raju, Structural engineering of polyurethane coatings for high performance applications, Progress in Polymer Science, vol 32, no 3, 2007, pp. 352-418, DOI: https:// doi.org/10.1016/j.progpolymsci.2006.05.003.

10. T. Park, S. So, B. Jeong, P. Zhou, J.-U. Lee, Life cycle assessment for enhanced Re-liquefaction systems applied to LNG carriers; effectiveness of partial Re-liquefaction system, Journal of Cleaner Production, vol 285, pp. 124832- 124853, 2021, , DOI: https://doi.org/10.1016/j.jclepro.2020.124832.

11. X. Chen, J. Chen, “Design of an arbitrary waveform signal generator", Procedia Engineering, vol 15, pp. 2500-2504, 2011. DOI: https://doi.org/10.1016/j.proeng.2011.08.470.

12. G. H. Castiblanco, W. Aperador. Equipo para la verificación de conexiones soldadas de tuberías recubiertas internamente, Revista De Investigación, Administración E Ingeniería Aibi vol:8, no. 3, pp. 60 - 66, DOI:10.15649/2346030X.867

13. G. Morettini, C. Braccesi, F. Cianetti, S.M.J. Razavi, “Design and implementation of new experimental multiaxial random fatigue tests on astm-a105 circular specimens", International Journal of Fatigue, vol 142, pp. 105983- 105996, 2021, DOI https://doi.org/10.1016/j.ijfatigue.2020.105983.

14. C. Li, Y. Song, A dummy cell added neural network using in pattern recognition for prevention of failed events, Microelectronics Journal, vol 68, pp. 23-31, 2017, , DOI: https://doi.org/10.1016/j. mejo.2017.08.013.

15. M. Ortega-Iguña, M. Chludzinski, C. Churiaque, R.E. Dos Santos, M. Porrúa-Lara, F. Abad-Fraga, J.M. Sánchez-Amaya, Mechanical behaviour of double side high performance PSA adhesive applied to painted naval structures, Polymer Testing, vol 93, pp. 106894- 106899, 2021, DOI: https://doi. org/10.1016/j.polymertesting.2020.106894.

16. M. Mobin, A.U. Malik, I.N. Andijani, F. Al-Muaili, M. Al-Hajri, G. Ozair, N.M.K. Mohammad, "Performance evaluation of some fusion-bonded epoxy coatings under water transmission line conditions", 
Progress in Organic Coatings, vol 62, no 4, pp. 369-375, 2008, DOI: https://doi.org/10.1016/j. porgcoat.2008.02.002.

17. Yuqing Bao, Jiming Gao, David T. Gawne, "Crack formation and its prevention in PVD films on epoxy coatings", Surface and Coatings Technology, vol 205, no. 1, pp. 15-21, 2010, DOI: https://doi. org/10.1016/j.surfcoat.2010.05.037.

18. M. Sababi, H. Terryn, J.M.C. Mol,"The influence of a Zr-based conversion treatment on interfacial bonding strength and stability of epoxy coated carbon steel", Progress in Organic Coatings, vol 105, pp. 29-36, 2017, DOI: https://doi.org/10.1016/j.porgcoat.2016.11.016.

19. Eli Leinov, Michael J.S. Lowe, Peter Cawley, Ultrasonic isolation of buried pipes,

20. Journal of Sound and Vibration, vol 363, pp. 225-239, 2016, DOI: https://doi.org/10.1016/j.jsv.2015.10.018.

21. K. Polo Bornachera., D.D. López Juvinao, and A. Henríquez Jaramillo, "Transferencia tecnológica para la producción limpia en la minería de materiales aluviales en La Guajira, Colombia," Investigación e Innovación en Ingenierías ., vol. 8, pp. 6-20, 2020. DOI: https://doi.org/10.17081/invinno.8.1.3535

22. C. Amaya, W. Aperador, J.C. Caicedo, F.J. Espinoza-Beltrán, J. Muñoz-Saldaña, G. Zambrano, P. Prieto, "Corrosion study of Alumina/Yttria-Stabilized Zirconia (Al2O3/YSZ) nanostructured Thermal Barrier Coatings (TBC) exposed to high temperature treatment", Corrosion Science, Volume 51, no 12, pp. 2994-2999, 2009, DOI: https://doi.org/10.1016/j.corsci.2009.08.028. 